\title{
Design and Modelling of Carbon Fiber Grid Structure based Carbon/Epoxy Composites for Enhanced Microwave Absorbing Properties
}

\author{
Aayushi Arya ${ }^{1, *}$, I. Srikanth ${ }^{2}$ \\ ${ }^{1}$ Department of Physics, NIT Kurukshetra 136118, Haryana, India \\ ${ }^{2}$ Advanced System Laboratory, DRDO, Kanchanbagh 500058, Hyderabad, India \\ *Corresponding author: E-mail: aayushi.arya1993@gmail.com
}

DOI: 10.5185/amlett.2020.111577

Frequency Tunable Microwave absorbers in desired low thickness is a critical requirement in electronics, radar and military applications where Electro Magnetic Interference has become a major concern. Composite are obvious choice of absorbers in above applications. The aim of this paper is to investigate the use of carbon fiber grid layers as Frequency Selective Surface (FSS) on Carbon Filler based Glass-Epoxy composites. In this work, three key points are highlighted, first is design, modeling of FSS grid, using equivalent circuit model and Transmission Line Theory, second is the use of fillers including Milled Carbon Fibers and Carbon Nano Tubes (CNTs) for microwave absorption, third is to have the desired reflection loss at specified thickness of around 6-7 $\mathrm{mm}$ which is low as compared to recent trends. It is found that the factors like the number of grid layers, their unit cell dimensions, type of filler, and the top surface layer, affects the resonance frequency and hence the microwave absorption characteristics of the composite laminates. The results are satisfactory showing minimum of -9.8 Db of Reflection Loss at 6-7 mm thickness laminates, for varied range of frequency.

\section{Introduction}

Electro Magnetic Interference has made Microwave absorbers an immediate requirement in various fields of electronic and military equipment [1]. To be an efficient microwave absorber, two conditions must be met, firstly its impedance should match with the free space impedance as shown in Fig. 1, second is the quick damping of the wave across the material. To achieve the latter condition, an absorption layer is created using Radar Absorbing materials(RAM), interchangeably called as microwave absorbing materials, which possess absorption of EM waves via intrinsic properties of the materials [2]. Through the years' resin Composites have emerged as convenient choice of absorbers. For the first condition, an additional impedance matching layer is to be added to the top of structure to trap the incident EM wave. This layer serves dual purpose of minimizing the reflection as well as providing frequency selectivity to the structure also known as Frequency Selective Surface [3]. Different materials absorb different frequency range. In this work, an attempt has been made to experimentally investigate the different aspects of microwave absorber including the radar absorbing layer and the FSS-grid layer. For the present Xband is chosen as the operational frequency, with focus on military and aerospace applications

\section{Radar Absorbing Material (RAM)}

As shown in Fig. 1(a) it consists of two components one is matrix and other are fillers. Matrix material provides damping to the incident microwave frequency as well as mechanical and structural strength to the composite while filler materials provides effective microwave absorption in the composite due to the polarization by the incident wave. Matrix is usually a high mechanical strength material example polymer resin which can be easily melded or given a shape [4].

The dielectric constant of the RAM grid structure is complex where the real part $\left(\varepsilon^{\prime}\right)$ represents the energy storage due to the polarization effect of the dipoles of the dielectric material [5]. The imaginary part $\left(\varepsilon^{\prime \prime}\right)$ represents the energy dissipation factor across the material which occurs due to change of orientation of the polar molecules to align along the applied electric field.

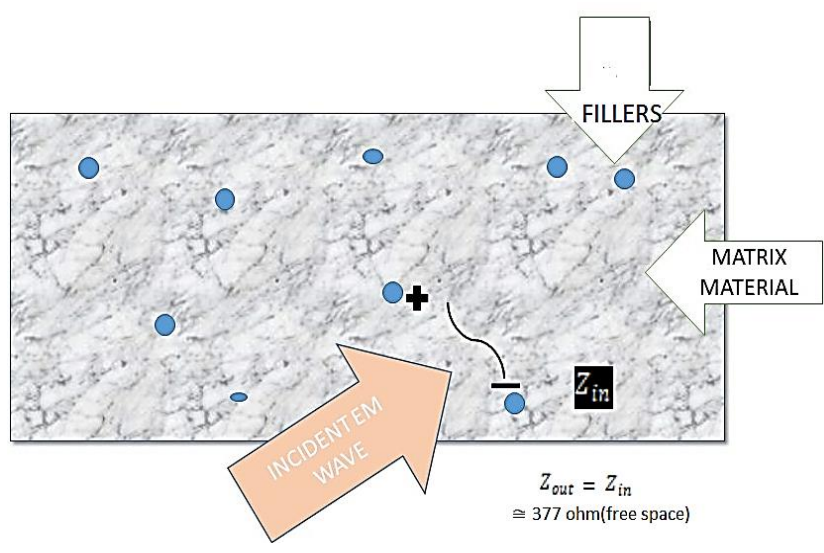

Fig. 1(a). Components of Microwave Absorber.

The introduction of filler particles alters the conductivity of the dielectric composite structure thereby 


\section{Advanced Materials Letters www.vbripress.com/aml}

altering the effective permittivity and loss tangent of the structure, as stated below

$$
\sigma=2 \pi f \varepsilon^{o} \epsilon^{\prime} \tan \delta
$$

where $\epsilon^{\prime}$ is the real part of the permittivity of the composite $f$ is the frequency, $\tan \delta$ is the loss tangent, $\sigma$ is the conductivity.

Complex permittivity of the composite allows them to behave as resonating structures. The real part of the permittivity depends on the glass fabric material, wherein the imaginary part depends on the filler particles, and the grid FSS layer. Glass Fiber Reinforced Plastics have emerged as a suitable candidate for composite applications in military and other core industries due to good mechanical, thermal and electrical properties [6]. Glass epoxy composites when added with carbon fillers acts as efficient microwave absorbers also.

\section{Fillers}

Fillers are conductive particles varying from micrometer range to nanometer scale which provides wave absorption to the composite structure. When the EM wave enters the composite, filler particles behaves as polarization hotspots allowing a formation of interconnected charged clusters, thereby creating dipole traps for the incident EM wave. Composite becomes a dielectric with the filler particles forming dielectric polar molecule. There is various polarization phenomenon occurring viz. interfacial polarization, multiple scattering, dipole formation, natural resonance. Fig. 1(b) depicts these phenomenon occurring in a RAM layer [7].

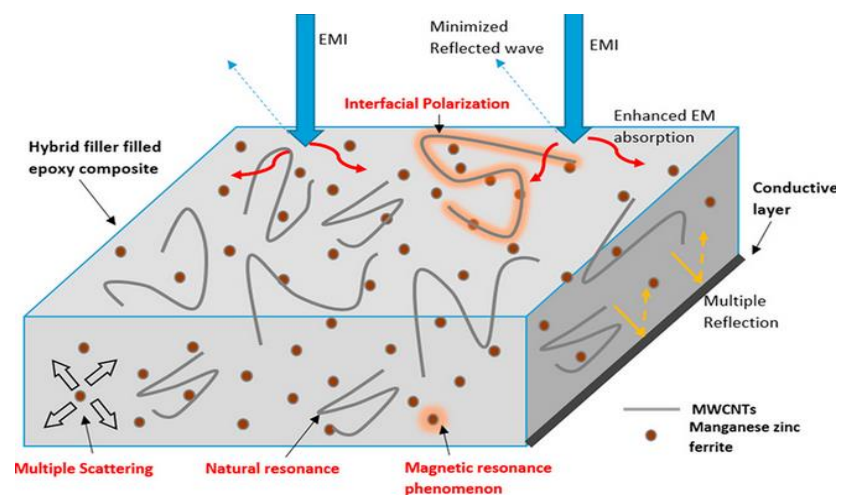

Fig. 1(b). Depicts these phenomenon occurring in a RAM layer [7].

Since the dispersion of the filler particles is not too dense and random, there polarization cause them to form random interconnectivity networks as similar to percolation networks, wherein they tend to occupy percolation sites of imaginary square lattice forming a conductive network of bonds as shown in Fig. 2(a) and Fig. 2(b) [8]. The conductive network acts as waveguide walls to the absorbed EM wave, which forms standing waves and further inhibits the transmission or reflection of the incident wave. The minimum volume fraction at which the onset of the network starts is called the percolation threshold. It depends on the geometry and the aspect ratio of the filler particle. Fig. 4 shows that with high aspect ratio there is enhanced interaction with the surrounding hence low percolation threshold, which is suitable requirement for high frequency range.

The physical effect can be seen with an increase in the effective permittivity of the structure by the introduction of the filler particle, hence increase in the Reflection Loss. The absorption by the filler particles depends on the shape, size, length, and the amount of filler particles. Altering the geometry of filler particles alters the interconnection network, thereby changing the absorption characteristics. We have investigated two types of filler one is Milled Carbon Fibers and other is Carbon Nano Tubes (CNTs) with high conductivity and high aspect ratio, which is more suitable at high frequency.

In this work we have opted for carbon based fillers, which also gives reinforcement to the matrix after blending. Changes in fillers like its content, geometry, composition can affectively alter the absorbing property $[\mathbf{1 , 2}]$.

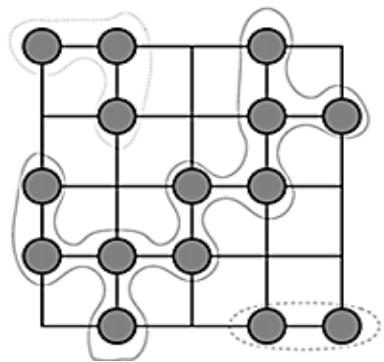

(a)

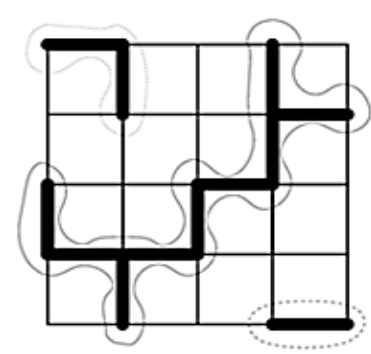

(b) (c)

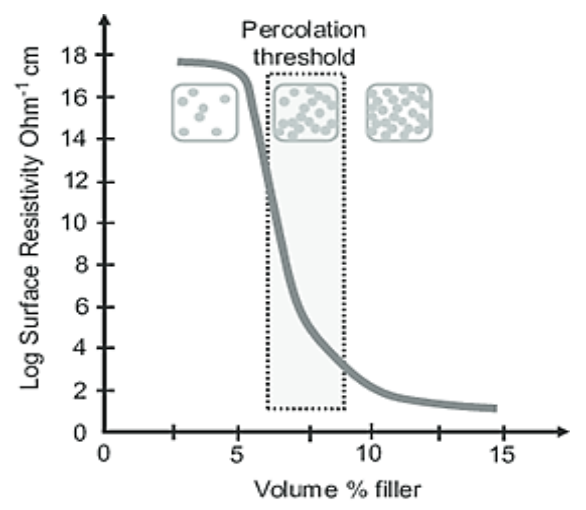

Fig. 2. (a) site percolation; (b) bond percolation [8]; (c) Variation of percolation threshold [9].

\section{Frequency selective surfaces}

Frequency selective surfaces are periodic geometrical patterns carved or lithographed on conductive surfaces, placed on top of a dielectric layer. The unit cell of the periodic structure acts as small resonating rings that filter outs the incident EM wave frequency, allowing only the resonant frequencies to pass. The patterns can be square ring, circular ring, rectangular slots, Jerusalem structures and so on. The dimension of the unit cells, periodicity, conductivity of the surface, permittivity and thickness of the dielectric layer FSS determine the resonant frequency. FSS are used to selectively either pass or reject a certain band of frequency. Thus they act as band pass or band stop 


\section{Advanced Materials Letters www.vbripress.com/aml}

filter. FSS structures works on resonance phenomenon. As depicted in Fig. 3(a), design of the FSS grid can alter the imaginary impedance of the composite to be either capacitive or inductive or a mix of the two. With the grid having more free transparent area, there is formation of capacitive network while more opaque area and transparent lines leads to formation of conducting lines across the composite structure to allow inductive magnetic effects to dominate [10]. These distributed capacitance and inductance causes attenuation of the incident Electric and Magnetic field respectively at the resonance range.

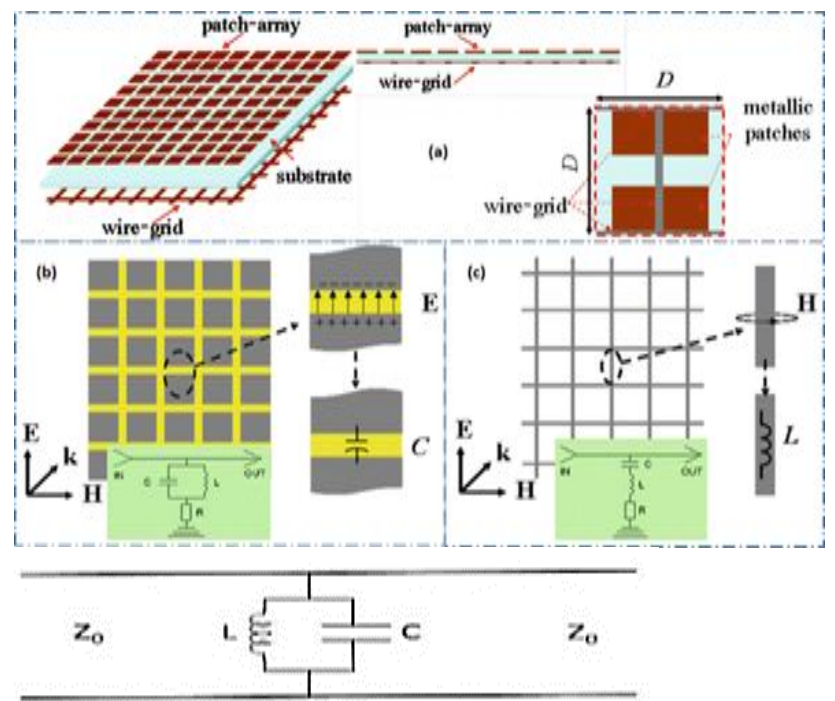

Fig. 3. (a) Reactive Components of Frequency Selective Surfaces [10]; (b) FSS equivalent band pass filter.

In recent years there is an extensive research to combine the FSS technique to the existing RAM layers forming a composite with increased input impedance [4]. The conventional designs of microwave absorbers were based on metallic FSS surfaces like Salisbury screens, jaumun absorbers that follow simple design rules of multiple reflection, phase cancellation due to the path difference created by the quarter wave layer. Although these designs were having tunable resonant frequency but due to heavy structures and low mechanical flexibility, became difficult to use in military applications. Carbon fiber grid structures emerged as an attracting alternative due to their good electrical conductivity esp. in the $\mathrm{GHz}$ range, good frequency selectivity and attenuation properties. Some of these efforts is summarized as follows. In [11] distributed resonant components were created by using carbon fiber felt screens acting as inductive element along with embedded vertically arranged carbon fibers acting introducing capacitance. The amplitude and phase of the EM wave was controlled by using quadrant technique, with two quadrant being inductive and other two capacitive. In [12] a gradient of impedance was created successfully using adjacent grid layers, which damped the EM wave in steps, with the first layer being a FSS layer. Similarly in [13] the effect of FSS created by periodic square patches of woven carbon fiber layer was observed in composites of PAN
(PolyAcrylonitrile) activated hollow porous Carbon Fibers (HPCF). There were also attempts to create multi layered composites by altering the impedance of each FSS layer. The preparation and study of such composites is described in [14] where an composite was designed for effective microwave absorption at wide oblique incident angles at a frequency range of around $5.8 \mathrm{GHz}$ particularly for Intelligent Transport System (ITS) application. The net impedance and reflection coefficient of the composite was calculated by accounting the effect of each layer. In this paper we designed a microwave absorber composite with epoxy resin and carbon-based fillers on top of which a carbon Fiber Grid structure was pasted which acts as a FSS layer. In The epoxy resin, which provided a structural base, we added carbon fillers like chopped carbon Fibers or MWCNTs. This creates a Radar Absorbing Layer (RAM). To this layer we paste a carbon Fiber grid layer which gives frequency selectivity, adjusting the peaks of the absorption characteristics. The grid was designed based on the Equivalent circuit model where the grid is modelled as a Band Pass Filter. We created single layer, Two layered and three layered structures with the order being firstly Carbon fiber filler, then MWCNT and again Carbon Fiber Filler respectively. The top grid was optimized to give maximum absorption by using the free space impedance matching principle. The composites were designed for various frequency bands. There was also an attempt of using carbon foam over the grid layer to maximize the EM wave incident on the grid layer. The composites were characterized using Vector Network Analyzer. The results appreciate their use in applications like EMI shielding where the equipments work in different frequency ranges like $5-6 \mathrm{GHz}$ and also for stealth based uses in military applications targeting 8-12 GHz range.

\section{Design of the composite microwave absorber}

\section{Grid design modelling}

The design of the grid structure is based on the transmission line theory and equivalent circuit model. The absorber is treated as a transmission line, with distributed resistance, capacitance across the length. The physical effects i.e. the losses, reflection can be modelled by equivalent circuit analysis with lumped parameters which can be calculated by characteristic impedance and absorption coefficient of the absorber. Each component of the absorber has been modeled as a lumped parameter.

The grid structure is modeled as a parallel LC resonance circuit which act as a bandpass filter, as shown in Fig. 3(b). Its equivalent grid structure and respective unit cell with ' $a$ ' being the length and ' $w$ ' combined width of the fiber sides as shown in Fig. 4(a) and Fig. 4(b) respectively.

Due to the grid design of the FSS layer, the ' $L$ ' component is lower as compared to the ' $\mathrm{C}$ ' component. Also the dielectric places below the FSS layer enhances the capacitive impedance, due to increase in effective permittivity. Thus, this LC circuit presents an FSS 


\section{Advanced Materials Letters www.vbripress.com/aml}

impedance $\left(Z_{F S S}\right)$ which is a complex function. This impedance is a complex function with its real part being the surface resistance $(R \sim 200-300 \mathrm{ohm})$ and imaginary part representing the inductive and capacitive effect. At resonance there is maximum impedance and thereby decreased value on either side of the peak resonating value. Other properties like - the whole calculation can be flexibly organized in below steps as follows:-

(i) FSS impedance $\left(Z_{F S S}\right)$, can be calculated by considering the required absorption coefficient $\tau$, which is calculated from the desired Reflection Loss (R.L).

(ii) Lumped capacitance, calculated from the Quality factor $(\mathrm{Q})$, which is a function of resonant frequency and surface resistance.

(iii) Unit cell dimensions ('a') can be extracted from the capacitance, with prior value of the permittivity of the grid layer material.

(iv) Radiation Parameters to derive the transverse wave mode impedances of the microwave absorbers.

(v) Finally the width of the unit cell, from the grid parameter.
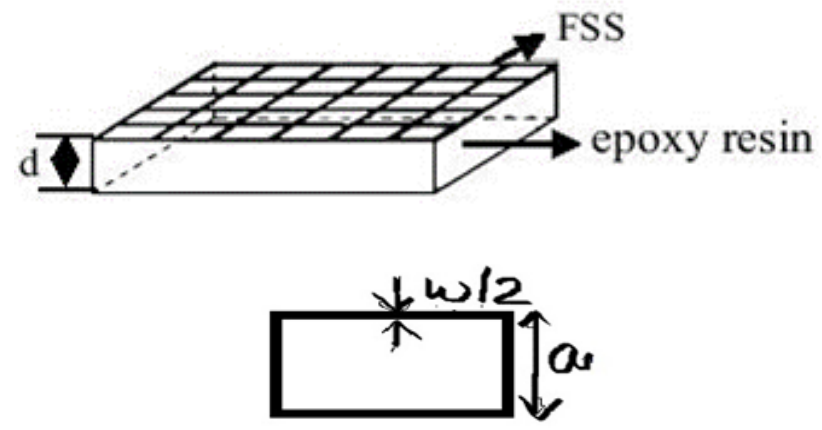

Fig. 4. (a) FSS with epoxy resin; (b) unit cell of grid.

Considering the case for maximum absorption, i.e. the characteristic impedance $Z_{o}$ of the absorber is equal to the free space impedance of $377 \Omega$, the initial parameters will be the required absorption, $\operatorname{coefficient}(\tau)$, FSS layer impedance $\left(Z_{F S S}\right)$, quality factor $(\mathrm{Q})$ which is a measure of the rate of attenuation, in this case below unity. It is expressed as the ratio of resonant frequency $\left(\omega_{o}\right)$ to the resonant frequency $\omega_{r}$. Other parameters required for the calculation are the lumped capacitance $\mathrm{C}$, permittivity of grid surface $\varepsilon_{r}$, unit cell aperture 'a', width of unit cell 'w', phase propagation constant of the wave $\beta$, transverse electric mode impedance of the absorber towards the EM wave $Z_{g}^{T E}$, effective intrinsic impedance of the wave $n_{e f f}$. Also, while determining the two dimensions of the unit cell through the equivalent circuit analysis, the grid parameter $(\alpha)$ also have its imposition on deciding the $\mathrm{a} / \mathrm{w}$ ratio. The grid parameter is the design aspect related to the attenuation of the electric field component along the surface of FSS.

In a multi grid layers design, there should be gradient of impedance wherein the impedance should increase as we go from top layer to the lower layer to minimize the surface reflection. Multi-layer design are suitable to achieve required absorption at low thickness, as it increases the effective impedance, thereby compensating the lowering of capacitive impedance due to the increase in permittivity at lower dielectric thickness.

Using the below sets of equations, we derive at certain sets of $\mathrm{w}$ and a for the grid structure e have formulated a chart based on the grid design and modelling as shown.

\section{Modelling equations}

\section{Experimental}

The Raw materials used for the matrix system are Diglycidyl ether of bisphenol A (DGEBA) epoxy resin and diethyl toluene diamine (DETDA) hardener. The two were mixed at a weight ratio of 100:24, and then manually mixed with specified weight ratio of Milled Carbon Fibers (MCFs). MCFs was processed from T-300 grade carbon fibers, which were chopped to $3 \mathrm{~mm}$ length and then fed to a chopping machine to create $500 \mu \mathrm{m}$. The chopped fibers were further ball milled at 250r/min (M/s Insmart Systems, India) for about half an hour to give fibers between 200-500 $\mu m$. Further S-glass layer was taken, applying the above mixture with a hand brush. Number of layers stacked are six in case of 2-layered FSS grid structure and three in case of three layered FSS grid structure. This gives a Radar absorbing Material layer (RAM) on top of these stacked layers the FSS grid layer, placed on S-glass, was pasted.

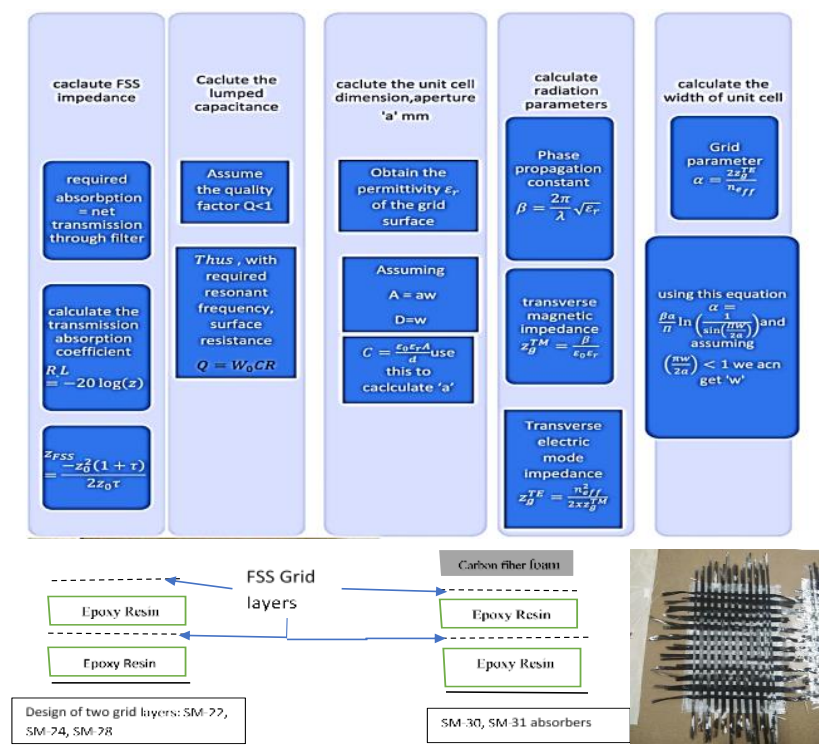

Fig. 5. (a) Mathematical modelling to calculate unit cell dimensions of the grid structure; (b) Design of grid layer embedded epoxy composites; (b)sample structure (c): grid layer.

This forms a complete grid-dielectric layer i.e. FSSRAM layer, which were repeated twice or thrice to form two layered FSS or three layered FSS structure respectively, followed by compaction to $6 \mathrm{~mm}$ using matched die molds. Further curing was carried at $100^{\circ} \mathrm{C}$ for 1 hour, $135^{\circ} \mathrm{C}$ for 3 hours. A set of samples were created to observe properties at different ratio and layers. In one sample FSS grid was placed on E-glass instead of S-glass. 


\section{Advanced Materials Letters www.vbripress.com/aml}

The data related to the samples is provided below. The table below shows the different samples and their structure, we have single FSS-grid sample SM-26, two layered structures SM-22, SM-24, SM-28, two with additional carbon black foam SM-30, SM-31 these were having Carbon fiber filler, SM-115, three layered structure, with Carbon fiber and carbon powder. SM-26, two layered structure with Carbon Nano Tubes. FSS unit cell dimension increasing as SM-24 $<$ SM-22 < SM-28, SM-26, SM-30< SM-115.

\section{Measurement}

The sample properties are measured in a Vector Network Analyzer (VNA) (M/s Keysight, Model PNA-N5224A), using free space measurement set up. Suitable fixtures are used to mount the samples steadily on a vibration free platform. After carrying out the Through-Reflect-Line calibration measurement for the reflection loss are taken. The samples are backed with a reflective conductor plate to avoid transmission. The whole frequency range was covered by using different adapter combinations which gives a good idea of near field reflection loss $\left(S_{11}\right)$. Out of them the average value is considered. The graph plot of R.L vs frequency are shown in Fig. 7.

\section{Results}

Table I. Sample structure with grid dimensions.

\begin{tabular}{|c|c|c|c|c|c|c|c|c|c|}
\hline $\begin{array}{l}\text { Sample } \\
\text {-Code }\end{array}$ & $\begin{array}{c}\text { TOP } \\
\text { COATIN } \\
\text { G }\end{array}$ & $\begin{array}{c}\text { FSS1 } \\
\text { A1(mm) } \\
\text { W1 }(\mathrm{mm})\end{array}$ & $\begin{array}{c}\text { FSS2 } \\
\text { A2 }(\mathrm{mm}) \\
\text { W2 }(\mathrm{mm})\end{array}$ & $\begin{array}{c}\text { FSS3 } \\
\text { A3(mm) } \\
\text { W3(mm) }\end{array}$ & GLASS & $\begin{array}{c}\mathrm{D} \text { (thickness } \\
\mathrm{mm})\end{array}$ & $\begin{array}{l}\text { Grid } \\
\text { layer }\end{array}$ & $\begin{array}{c}\text { FILLER } \\
\text { CONTENT }\end{array}$ & $\begin{array}{l}\text { Total } \\
\text { Thicknes } \\
\text { s-mm }\end{array}$ \\
\hline SM-22 & - & $\begin{array}{l}20 \\
10\end{array}$ & $\begin{array}{c}14 \\
7\end{array}$ & - & S-GLASS & 2.5 & 2 & $\mathrm{CF}(0.43 \%)$ & $\begin{array}{c}200 \times 200 \\
\times 6\end{array}$ \\
\hline SM-24 & - & $\begin{array}{c}18 \\
9\end{array}$ & $\begin{array}{c}12 \\
6\end{array}$ & -- & S-GLASS & 2.5 & 2 & CF(0.43\%) & $\begin{array}{l}200 \times 200 \\
\times 6\end{array}$ \\
\hline SM-28 & - & $\begin{array}{l}22 \\
11\end{array}$ & $\begin{array}{c}16 \\
8\end{array}$ & - & $\begin{array}{r}\text { S- } \\
\text { GLASS }\end{array}$ & 2.5 & 2 & CF(0.43wt\%) & $\begin{array}{c}200 \times 200 \\
\times 6\end{array}$ \\
\hline SM-30 & $\begin{array}{l}\text { Black } \\
\text { Foam }\end{array}$ & $\begin{array}{l}22 \\
11\end{array}$ & $\begin{array}{c}16 \\
8\end{array}$ & - & $\begin{array}{c}\text { S- } \\
\text { GLASS }\end{array}$ & 2.5 & 2 & CF(0.43\%) & $\begin{array}{l}200 \times 200 \\
\times 6\end{array}$ \\
\hline SM-31 & $\begin{array}{l}\text { Black } \\
\text { Foam }\end{array}$ & $\begin{array}{l}22 \\
11\end{array}$ & $\begin{array}{l}16 \\
18\end{array}$ & -- & S-GLASS & 2.5 & 2 & CF(0.43\%) & $\begin{array}{l}200 \times 200 \\
\times 6\end{array}$ \\
\hline SM-26 & - & $\begin{array}{l}22 \\
11\end{array}$ & : & -- & S-GLASS & 5.0 & 1 & CNT( 5 wt\%) & $\begin{array}{l}200 \times 200 \\
\times 6\end{array}$ \\
\hline SM-115 & -. & $\begin{array}{l}23 \\
17\end{array}$ & $\begin{array}{l}21 \\
13\end{array}$ & $\begin{array}{l}19 \\
11\end{array}$ & E-GLASS & 6.6 & 3 & $\begin{array}{l}\text { CF }(0.7 w t \%) \\
\text { CP(1.2wt\%) }\end{array}$ & $\begin{array}{l}250 \times 250 \\
\times 7\end{array}$ \\
\hline
\end{tabular}

Table II. Performance of various samples based on absorption peak and bandwidth at half power.

\begin{tabular}{llll}
\hline Sample & $\begin{array}{l}\text { Absorption } \\
\text { peak }\end{array}$ & Frequency & $\mathbf{- 5 ~ d B ~ b a n d w i d t h ~}$ \\
\hline $\mathrm{SM}-22$ & $-9.8 \mathrm{Db}$ & $6.5 \mathrm{GHz}$ & $5.8-6.5 \mathrm{GHz}(0.7 \mathrm{GHz})$ \\
$\mathrm{SM}-24$ & $-9.88 \mathrm{Db}$ & $8.3 \mathrm{GHz}$ & $5.8-8.3 \mathrm{GHz}(2.5 \mathrm{GHz})$ \\
$\mathrm{SM}-28$ & $\mathrm{NIL}$ & $\mathrm{NIL}$ & $\mathrm{NIL}$ \\
$\mathrm{SM}-30$ & $-9.42 \mathrm{Db}$ & $5.8 \mathrm{GHz}$ & $\mathrm{Nil}$ \\
$\mathrm{SM}-31$ & $-9 \mathrm{Db}$ & $18 \mathrm{GHz}$ & $12.2-18 \mathrm{GHz}(6 \mathrm{GHz})$ \\
$\mathrm{SM}-26$ & $-9.8 \mathrm{Db}$ & $13.6 \mathrm{GHz}$ & $7.0-13 \mathrm{GHz}(6 \mathrm{GHz})$ \\
$\mathrm{SM}-115$ & $-9.8 \mathrm{Db}$ & $4.3 \mathrm{GHz}$, & Two peaks bandwidth: \\
& & $12 \mathrm{GHz}$ & $4-4.5 \mathrm{GHz}(0.5 \mathrm{GHz})$ \\
& & & $11-13 \mathrm{GHz}(3 \mathrm{GHz})$ \\
\hline
\end{tabular}

\section{Graph plots}

Based on the above results, it can be seen that as we increase the number of FSS-grid layers that is from single to multiple ,the absorption tends to be broadband and the resonance peak shifts towards lower range of frequency (between 5-10 GHz). For the three-layered structure we have obtained a wide bandwidth but with absorption peak at two frequencies one at lower and another in $\mathrm{X}$ band (4 GHz, $12 \mathrm{GHz}$ ). Similarly increasing the unit cell dimensions, causes the resonance frequency to shift to a lower frequency. It can be because of the increased wavelength, scattered by larger square grid. However for the composite to work at much lower frequency below $5 \mathrm{GHz}$, the dielectric constant of the RAM layer should be increased to support the reduced relaxation time constant of polar molecules. Also use of carbon black foam as top layer can support the lower frequency capturing. And for the FSS to work at proper resonance frequency it should be kept above the RAM layer. As per the filler particles Carbon fiber fillers works good in the frequency range of 5-14 Ghz but MWCNTs can give good performance for much higher frequencies of 8-18 Ghz and give more broadband performance. This can be attributed to the high conductivity and high aspect ratio of CNTs. The results shows agreement with the theory discussed in above section.
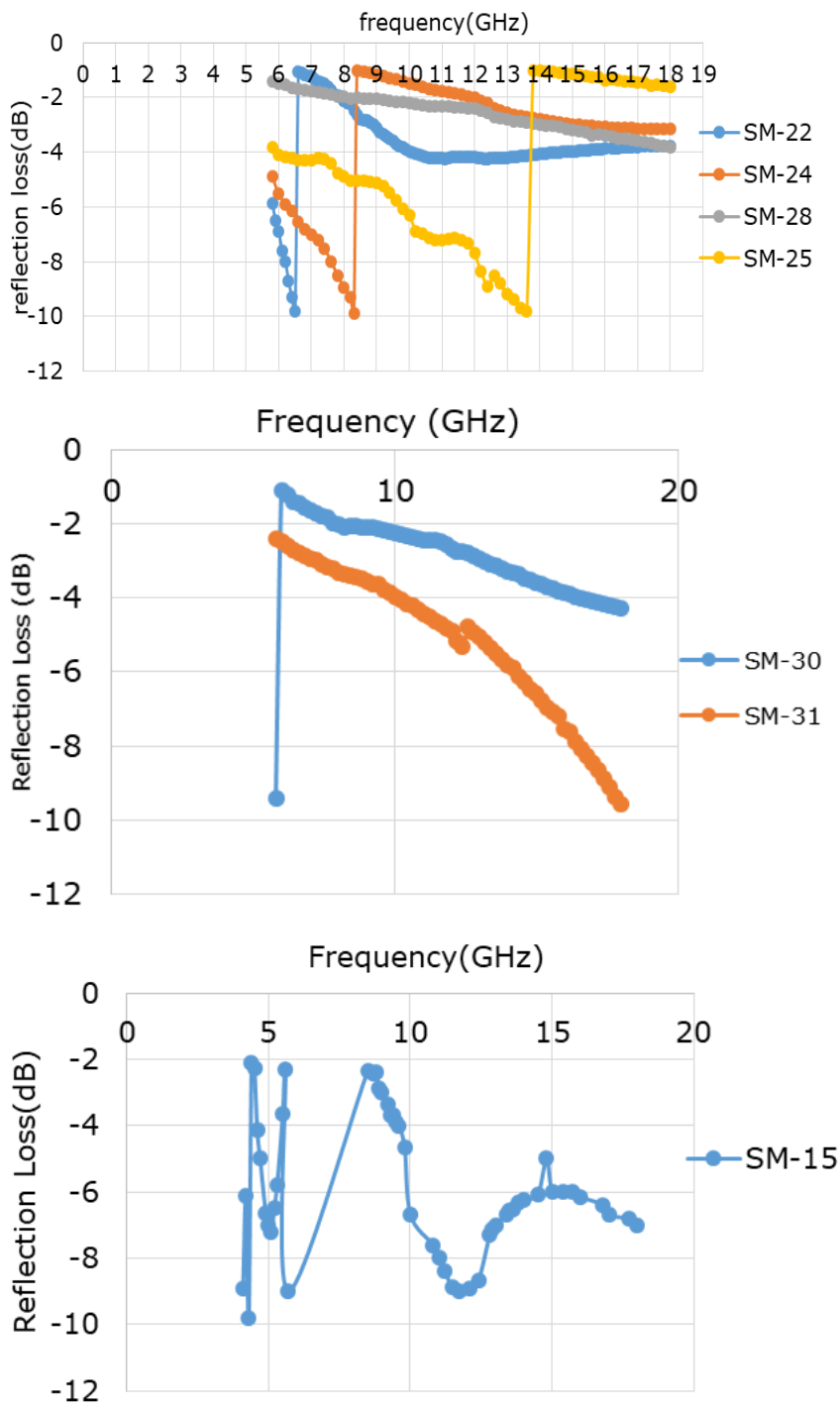

Fig. 7. (a) result for two-layer samples; (b) result for foam samples (c) result for the three sample. 


\section{Advanced Materials Letters www.vbripress.com/aml}

\section{Conclusion}

The above results prove that using FSS grid layer not only provides a way of tuning and adjusting the resonance peaks of the microwave composite laminate but also provides a way to fabricate low thickness laminates which can be used for various applications. The work benefits to give ideas to be able to modify conventional composite structure at desirable frequencies, just by adding FSS-grid layer and changing filler content. Based on above conclusion a broadband composite can be designed as a multi grid layered structure with the above RAM layer to absorb lower frequency and the below RAM working at Higher Frequency and their FSS layer resonating at their respective frequencies. This work can be useful for applications like military stealth aircrafts, Electro Magnetic Shielding for electronic equipment's, wireless mobile communications, wireless transport communication, near future sensor based automotive vehicles. Also it can be useful for medical industry to isolate critical patient chambers from EM exposure. This technology can be integrated with wall paints to form special EM resistant buildings.

\section{Acknowledgments}

The authors gratefully acknowledge to the Advanced Systems Laboratory, DRDO, Hyderabad for their support, guidance and for providing necessary resources for the fabrication of the samples.

\section{Conflict of interests}

The author(s) declared no potential conflicts of interest with respect to the research, authorship, and/or publication of this article.

\section{Keywords}

Glass epoxy composites, frequency selective surfaces, carbon fiber grid, modelling circuit, VNA.

\section{References}

1. Ibrahim, I.R.; Matori, K.A.; Ismail, I.; et al. Sci. Rep., 2020, 10, 3135.

2. Ki-Yeon Park, Jae-Hung Han, Sang-Bok Lee et al. Composites Science and Technology, 2009, 69, 1271.

3. Lee, J.; Yoo, M.; Lim, S.; IEEE Transactions on Antennas and Propagation, 2014, 63, 221

4. Liu, X.; Zhang, Z.; Wu, Y.; Composites Part B: Engineering, 2011, 42, 326.

5. Kim, J.B.; Lee, S.K.; Kim, C.G.; Composites Science and Technology, 2008, 68, 2909.

6. Ivaturi, S.; Baske, H.; Ghosal, P.; Journal of Composite Materials, 2019, 53, 2891.

7. Phan, C.H.; Mariatti, M.; Koh, Y.H.; Journal of Magnetism and Magnetic Materials, 2016, 401, 472.

8. Zare, Y.; Rhee, K.Y.; Polymers, 2020, 12, 182.

9. DeArmitt, Chris.; Applied Plastics Engineering Handbook, 2011

10. Tong, X.C., Metamaterials Inspired Frequency Selective Surfaces. In Functional Metamaterials and Metadevices, Springer, Cham, 2018, pp.155-171.

11. Qin, F.; Brosseau, C.; Journal of Applied Physics, 2012, 111, 061301.

12. Neo, C.P.; Vardhan, Vijay K.; IEEE Transactions on Electromagnetic Compatibility, 2004, 46.

13. Zhao, Naiqin; Zou, Tianchum; Shi, Chunsheng; et al., Material Science and Engineering B., 2006, 207.

14. Zhang, Jun; Xiao, Peng; Zhou, Wei; et al., J. Mater Sci: Mater Electron, 2014.

15. Xie, Wei; Cheng, Hai-Feng; Chu, Zeng-Yong; et al., Material and Design, 2009, 30, 1201.
16. Jae-Woong Kim, Sung-Soo Kim, Materials and Design, 2010, 1547.

17. Shaoxu Xia, Bin Yao, qiping Chen et al., Composites part B, 2016,

18. Yongju Zang, Shaoxu Xia, Liwei Li et al., Composite part B, 2015, 371.

19. Lee, W.J.; Lee, J.W.; Kim, C.G.; Microwave Absorbing Structure with Conducting Polymer FSS Coating, $16^{\text {th }}$ International Conference on Composite Materials.

20. Filippo Costa, Agostina Monorchio; IEEE Transactions on Antennas and propagation, 2012, 60, 2740.

21. Meng Zhang, Tian Jiang, Yijun Feng; Journal of Electromagnetic Analysis and Applications, 2014, 203.

\section{Authors biography}

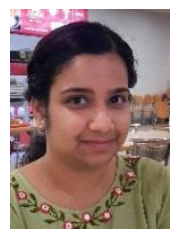

Aayushi Arya M.Tech Dept. of Physics NIT Kurukshetra, pursuing Phd IIT Hyderabad. Area of interest include EM wave properties in different materials, Microwave absorption, theoretical and mathematical analysis of absorbing materials.

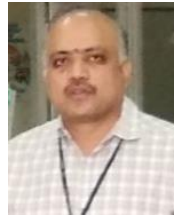

Dr. Srikanth Ivaturi, Phd IIT Hyderabad Presently working as Scientist 'E' at Advanced Systems Laboratory, Hyderabad. His areas of interest includes: Radar absorbing structures, ultra high temperature ceramics, and nano material added CFRP/GFRPs.

\section{Graphical abstract}

Glass-Epoxy composite laminate with FSS grid pattern structure

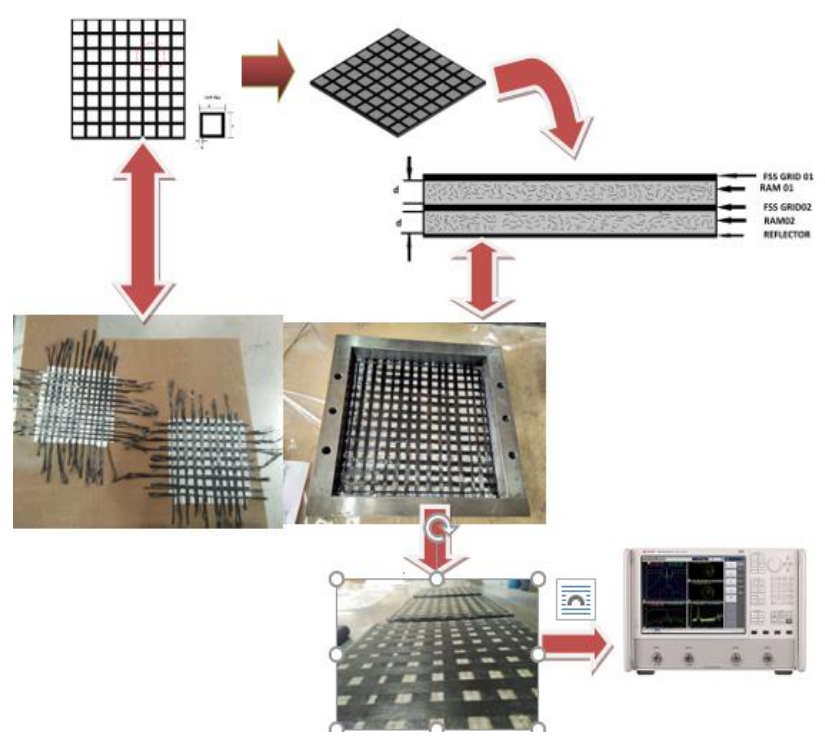

